\title{
Generalized bipolar product and sum
}

\author{
Salvatore Greco ${ }^{1}$, Radko Mesiar $^{2}$, and Fabio Rindone ${ }^{3 \star}$ \\ 1 Department of Economics and Business \\ 95029 Catania, Italy \\ and
}

University of Portsmouth, Portsmouth Business School, Centre of Operations Research and Logistics (CORL), Richmond Building, Portland Street, Portsmouth PO1 3DE, United Kingdom salgreco@unict.it

2 Department of Mathematics and Descriptive Geometry, Faculty of Civil Engineering

Slovak University of Technology

Bratislava, Slovakia

and

Institute for Research and Applications of Fuzzy Modeling

Division of University Ostrava

NSC IT4Innovations

30. dubna 22, Ostrava 70103

Czech Republic

radko.mesiar@stuba.sk

3 Department of Economics and Business

95029 Catania, Italy

frindone@unict.it

\begin{abstract}
Aggregation functions on $[0,1]$ with annihilator 0 can be seen as a generalized product on $[0,1]$. We study the generalized product on the bipolar scale $[-1,1]$, stressing the axiomatic point of view ( compare also [9]). Based on newly introduced bipolar properties, such as the bipolar increasingness, bipolar unit element, bipolar idempotent element, several kinds of generalized bipolar product are introduced and studied. A special stress is put on bipolar semicopulas, bipolar quasi-copulas and bipolar copulas. Inspired by the truncated sum on $[-1,1]$ we introduce also the class of generalized bipolar sums, which differ from uninorms due to the non-associativity.
\end{abstract}

Keywords: Aggregation function; bipolar copula; bipolar scale; bipolar semicopula; symmetric minimum.

\section{Introduction}

Recall that an aggregation function $A:[0,1]^{2} \rightarrow[0,1]$ is characterized by boundary conditions $A(0,0)=0$ and $A(1,1)=1$, and by the increasingness of $A$ (we use the term increasing in the weak form, meaning that $x<y$ implies $f(x) \leq f(y)$ ), i.e., the sections $A(x, \cdot)$ and $A(\cdot, y)$ are increasing for each $x, y \in[0,1]$. For more details see [2,

\footnotetext{
* Corresponding author. E-mail: frindone@ unict.it
} 
7]. The product $\Pi:[0,1]^{2} \rightarrow[0,1]$ has additionally 0 as its annihilator, and thus each aggregation function $A:[0,1]^{2} \rightarrow[0,1]$ with annihilator 0 (i.e., $A(x, 0)=A(0, y)=0$ for all $x, y \in[0,1])$ can be seen as a generalization of the product $\Pi$ on the unipolar scale $[0,1]$. Observe that the class $\mathcal{P}$ of generalized products on the scale $[0,1]$ has the smallest aggregation function $A_{*}:[0,1]^{2} \rightarrow[0,1]$ given by

$$
A_{*}(x, y)=\left\{\begin{array}{l}
1 \text { if } x=y=1 \\
0 \text { else }
\end{array}\right.
$$

as its minimal element, and its maximal element $A^{*}:[0,1]^{2} \rightarrow[0,1]$ is given by

$$
A^{*}(x, y)=\left\{\begin{array}{l}
0 \text { if } x y=0 \\
1 \text { else }
\end{array}\right.
$$

Convex sums of these extremal generalized products, give rise to a parametric family connecting them.

Moreover $\mathcal{P}$ is a complete lattice (with respect to pointwise suprema and infima), and it contains, among others, geometric mean $G$, harmonic mean $H$, minimum $M$, etc. The most distinguished subclasses of $\mathcal{P}$ are

- the class $\mathcal{S}$ of semicopulas, i.e., aggregation functions from $\mathcal{P}$ having $e=1$ as neutral element, $S(x, 1)=S(1, x)=x$ for all $x \in[0,1]$, see $[1,4]$;

- the class $\mathcal{T}$ of triangular norms, i.e., of associative and commutative semicopulas, $[15,19]$;

- the class $\mathcal{Q}$ of quasi-copulas, i.e. 1 -Lipschitz aggregation functions from $\mathcal{P}$ (observe that $\mathcal{Q} \subsetneq \mathcal{S})$, [18];

- the class $\mathcal{C}$ of copulas, i.e. supermodular functions from $\mathcal{P}$ (observe that $\mathcal{C} \subsetneq \mathcal{Q}$ ), [18].

Recall that a real function $B:[a, b]^{2} \rightarrow \mathbb{R}$ is 1 -Lipschitz (supermodular), whenever, for each $\left(x_{1}, x_{2}\right),\left(x_{2}, y_{2}\right) \in[a, b]^{2}$, it holds

$$
\begin{gathered}
\left|B\left(x_{1}, x_{2}\right)-B\left(x_{2}, y_{2}\right)\right| \leq\left|x_{1}-x_{2}\right|+\left|y_{1}-y_{2}\right| \\
{\left[B\left(x_{1} \vee x_{2}, y_{1} \vee y_{2}\right)+B\left(x_{1} \wedge x_{2}, y_{1} \wedge y_{2}\right) \geq B\left(x_{1}, y_{1}\right)+B\left(x_{2}, y_{2}\right) .\right]}
\end{gathered}
$$

Observe that the product $\Pi$ belongs to any of mentioned classes, similarly as $M$. Among several applications of the generalized product functions, recall their role as conjunctions in fuzzy logic [13], or their role of multiplications in the area of general integrals $[16,21]$.

Integration on bipolar scale $[-1,1]$ requires a bipolar function $B:[-1,1]^{2} \rightarrow$ $[-1,1]$ related to the standard product $\Pi:[-1,1]^{2} \rightarrow[-1,1]$ (we will use the same notation $\Pi$ for the product independently of the actual scale). Up to standard product $\Pi$ applied, e.g., in the case of Choquet integral on $[-1,1]$, or in the case of bipolar capacities based Choquet integral, Grabisch [5] has introduced a symmetric Sugeno integral on $[-1,1]$ based on the symmetric minimum $B_{M}:[-1,1]^{2} \rightarrow[-1,1], B_{M}(x, y)=$ $\operatorname{sign}(x y) \min (|x|,|y|)$. Moreover, Greco and Rindone [11] have introduced the bipolar Sugeno integral, again based on the symmetric minimum $B_{M}$. The aim of this paper 
is to generalize the bipolar product on $[-1,1]$ in a way similar to generalized product on $[0,1]$, and to study special classes of such generalizations. Clearly, the idea to study generalized products stems from bipolar integrals. In turn, bipolar integrals are inserted in the rich domain of bipolarity.A wide literature, developed in very recent years, has demonstrated how bipolarity must be considered in modeling human reasoning ${ }^{4}$.

The paper is organized as follows. In the next section, several properties of bipolar functions are proposed, and the generalized bipolar product is introduced. Section 3 is devoted to bipolar semicopulas and bipolar triangular norms, while in Section 4 we study bipolar quasi-copulas and bipolar copulas. Finally, some concluding remarks are added.

\section{Generalized bipolar product}

Considering the function $F:[-1,1]^{2} \rightarrow[-1,1]$, several algebraic and analytic properties can be considered in their standard form, such as the commutativity, associativity, annihilator 0, continuity, Lipschitzianity, supermodularity, etc. Note that the bipolar product $\Pi:[-1,1]^{2} \rightarrow[-1,1]$ as well as the symmetric minimum $B_{M}:[-1,1]^{2} \rightarrow$ $[-1,1]$ satisfy all of them. However, there are some properties reflecting the bipolarity of the scale $[-1,1]$.

Recall that a mapping $S:[0,1]^{2} \rightarrow[0,1]$ is a semicopula [1,4], whenever it is increasing in both variables and 1 is the neutral element, i.e., $S(x, 1)=S(1, x)=x$ for all $x \in[0,1]$. When considering the product $\Pi:[-1,1]^{2} \rightarrow[-1,1]$, we see that 1 is its neutral element. More, it holds $\Pi(-1, x)=\Pi(x,-1)=-x$ for all $x \in[-1,1]$.

Definition 1 Let $F:[-1,1]^{2} \rightarrow[-1,1]$ be a mapping such that $F(x, 1)=F(1, x)=$ $x$ and $F(-1, x)=F(x,-1)=-x$ for all $x \in[-1,1]$. Then 1 is called a bipolar neutral element for $F$.

Simple bipolar semicopulas $B_{S}$, introduced for bipolar universal integrals in [8], are fully determined by standard semicopulas $S:[0,1]^{2} \rightarrow[0,1]$, by means of $B_{S}(x, y)=$ $(\operatorname{sign}(x y)) S(|x|,|y|)$. Observe that 1 is a bipolar neutral element for any simple bipolar semicopula $B_{S}$. Concerning the monotonicity required for semicopulas, observe that considering the product $\Pi$, or any simple bipolar semicopula $B_{S}$ (note that $B_{\Pi}=\Pi$, abusing the notation $\Pi$ both for the product on $[-1,1]$ and on $[0,1])$, these mappings are increasing in both coordinates when fixing an element from the positive part of the scale, while they are decreasing when fixing an element from the negative part of the scale $[-1,1]$.

Definition 2 Let $F:[-1,1]^{2} \rightarrow[-1,1]$ be a mapping such that the partial mappings $F(x, \cdot)$ and $F(\cdot, y)$ are increasing for any $x, y \in[0,1]$ and they are decreasing for any $x, y \in[-1,0]$. Then $F$ will be called a bipolar increasing mapping.

\footnotetext{
${ }^{4}$ In decision theory bipolarity has firstly been modeled by Tversky and Kahneman [14, 20] (see also [12]). The reader is referred to [3] for a general treatment of bipolarity, and to [6] for discussion of bipolar and bivariate models in a multiple criteria framework.
} 
Similarly, inspired by the symmetric minimum $B_{M}$, we introduce the notion of a bipolar idem potent element.

Definition 3 Let $F:[-1,1]^{2} \rightarrow[-1,1]$ be given. An element $x \in[0,1]$ is called $a$ bipolar idempotent element of $F$ whenever it satisfies $F(x, x)=F(-x,-x)=x$ and $F(-x, x)=F(x,-x)=-x$.

Recall that the class $\mathcal{P}$ of generalized products on $[0,1]$ can be characterized as the class of all the increasing mappings $\left.F:[0,1]^{2} \rightarrow 0,1\right]$ such that $\left.F\right|_{\{0,1\}^{2}}=\left.\Pi\right|_{\{0,1\}^{2}}$. Inspired by this characterization, we introduce the class $\mathcal{B P}$ of all generalized bipolar products as follows.

Definition 4 A function $B:[-1,1]^{2} \rightarrow[-1,1]$ is a generalized bipolar product whenever it is bipolar increasing and $\left.B\right|_{\{-1,0,1\}^{2}}=\left.\Pi\right|_{\{-1,0,1\}^{2}}$.

Theorem $1 B \in \mathcal{B P}$ if and only if there are $A_{1}, A_{2}, A_{3}, A_{4} \in \mathcal{P}$ such that

$$
B(x, y)=\left\{\begin{array}{lll}
A_{1}(x, y) & \text { if } \quad(x, y) \in[0,1]^{2} \\
-A_{2}(-x, y) & \text { if } \quad(x, y) \in[-1,0] \times[0,1] \\
A_{3}(-x,-y) & \text { if } \quad(x, y) \in[-1,0]^{2} \\
-A_{4}(x,-y) & \text { if } \quad(x, y) \in[0,1] \times[-1,0] .
\end{array}\right.
$$

Proof. The necessity can be checked considering the properties of restrictions $\left.B\right|_{[0,1]^{2}}$, $\left.B\right|_{[-1,0]^{2}},\left.B\right|_{[-1,0] \times[0,1]}$ and $\left.B\right|_{[0,1] \times[-1,0]}$. The sufficiency is a matter of verifying the bipolar monotonicity of $B$ and the equality $\left.B\right|_{\{-1,0,1\}^{2}}=\left.\Pi\right|_{\{-1,0,1\}^{2}}$. These properties can be checked by cases. For example $B(-1,-1)=A_{3}(1,1)=\Pi(1,1)=1$.

Due to Theorem 1, each $B \in \mathcal{B P}$ can be identified with a quadruple $\left(A_{1}, A_{2}, A_{3}, A_{4}\right) \in$ $\mathcal{P}^{4}$.

Definition 5 Let $A \in \mathcal{P}$. then $B_{A}=(A, A, A, A) \in \mathcal{B P}$, given by $B_{A}(x, y)=$ $\operatorname{sign}(x y) A(|x|,|y|)$, is called a simple generalized bipolar product (simple GBP, in short).

Evidently, $B_{M}$ is a simple $G B P$ related to $M$, while $B_{\Pi}=\Pi$. Observe that

$$
B_{A_{*}}(x, y)= \begin{cases}\Pi(x, y) & \text { if }(x, y) \in\{-1,1\} \\ 0 & \text { else, }\end{cases}
$$

and

$$
B_{A^{*}}(x, y)=\operatorname{sign}(x y) .
$$

However $B_{A_{*}}$ and $B_{A^{*}}$ are not extremal elements of $\mathcal{B P}$. The class $\mathcal{B P}$ is a complete lattice (considering pointwise sup and inf) with top element $B^{*}=\left(A^{*}, A_{*}, A^{*}, A_{*}\right)$ and bottom element $B_{*}=\left(A_{*}, A^{*}, A_{*}, A^{*}\right)$, given by 


$$
B^{*}(x, y)= \begin{cases}1 & \text { if } \quad x y>0 \\ -1 & \text { if } \quad x y=-1 \\ 0 & \text { else, }\end{cases}
$$

and

$$
B_{*}(x, y)= \begin{cases}-1 & \text { if } \quad x y<0 \\ 1 & \text { if } x y=1 \\ 0 & \text { else. }\end{cases}
$$

As in the case of generalized products on the scale $[0,1]$, to obtain a parametric family connecting $B_{*}$ and $B^{*}$ it is enough to consider their convex sum.

\section{Bipolar semicopulas and bipolar t-norms}

Based on the idea of a bipolar neutral element $e=1$, we introduce now the bipolar semicopulas, compare also [10].

Definition 6 A mapping $B:[-1,1]^{2} \rightarrow[-1,1]$ is called a bipolar semicopula whenever it is bipolar increasing and 1 is a bipolar neutral element of $B$.

Based on Theorem 1 we have the next result

Corollary 1 A mapping $B:[-1,1]^{2} \rightarrow[-1,1]$ is a bipolar semicopula if and only if there is a quadruple $\left(S_{1}, S_{2}, S_{3}, S_{4}\right)$ of semicopulas so that

$$
B(x, y)=\left\{\begin{array}{lll}
S_{1}(x, y) & \text { if } & (x, y) \in[0,1]^{2} \\
-S_{2}(-x, y) & \text { if } & (x, y) \in[-1,0] \times[0,1] \\
S_{3}(-x,-y) & \text { if } & (x, y) \in[-1,0]^{2} \\
-S_{4}(x,-y) & \text { if } & (x, y) \in[0,1] \times[-1,0]
\end{array}\right.
$$

It is not difficult to check that the extremal bipolar semicopulas are related to extremal semicopulas $M$ (the greatest semicopula given by $M(x, y)=\min (x, y))$ and $Z$ (the smallest semicopula, called also the drastic product, and given by $Z(x, y)=$ $\min (x, y)$ if $1 \in\{x, y\}$ and $Z(x, y)=0$ else). For details see [10, Proposition 1].

We have also the next results.

Proposition 1 [10] Let $B \in \mathcal{B}$ be a bipolar semicopula such that each $x \in[0,1]$ is its bipolar idempotent element. Then $B=B_{M}$ is the symmetric minimum introduced by Grabisch [5].

Associativity of binary operations (binary functions) is a strong algebraic property, which, in the case of bipolar semicopulas characterizes a particular subclass of $\mathcal{B}$.

Theorem 2 [10] A bipolar semicopula $B \in \mathcal{B}$ is associative if and only if $B$ is a simple bipolar semicopula, $B=B_{S}$, where $S \in \mathcal{S}$ is an associative semicopula. 
Typical examples of associative bipolar semicopulas are the product $\Pi$ and the symmetric minimum $B_{M}$. Recall that a symmetric semicopula $S \in \mathcal{S}$, i.e., $S(x, y)=$ $S(y, x)$ for all $x, y \in[0,1]$, which is also associative is called a triangular norm [19, $15]$.

Definition 7 A symmetric associative bipolar semicopula $B \in \mathcal{B}$ is called a bipolar triangular norm.

Due to Theorem 2 it is obvious that a bipolar semicopula $B \in \mathcal{B}$ is a bipolar triangular norm if and only if $B=B_{T}$, where $T:[0,1]^{2} \rightarrow[0,1]$ is a triangular norm, i.e. if $B(x, y)=(\operatorname{sign}(x y)) T(|x|,|y|)$. Obviously, the product, $\Pi$, and the symmetric minimum, $B_{M}$, are bipolar triangular norms. The smallest semicopula $Z$ is also a triangular norm and the corresponding bipolar triangular norm $B_{Z}:[-1,1]^{2} \rightarrow[-1,1]$ is given by

$$
B_{Z}(x, y)= \begin{cases}0 & \text { if }(x, y) \in]-1,1\left[^{2}\right. \\ x y & \text { else }\end{cases}
$$

Observe that due to the associativity of $B_{Z}$ there is unique distinguished n-ary extension (often called "genuine"), $B_{Z}:[-1,1]^{n} \rightarrow[-1,1], n>2$ given by

$$
B_{Z}\left(x_{1}, \ldots, x_{n}\right)= \begin{cases}0 & \text { if } \#\left\{i \mid x_{i} \in\right]-1,1[\} \geq 2 \\ \prod_{i=1}^{n} x_{i} & \text { else. }\end{cases}
$$

Also $W$ is a triangular norm, and thus also $B_{W}:[-1,1]^{2} \rightarrow[-1,1]$ given by $B_{W}(x, y)=(\operatorname{sign}(x y)) \max (0, x+y-1)$ is a bipolar triangular norm. Moreover, its $\mathrm{n}$-ary extension $B_{W}:[-1,1]^{n} \rightarrow[-1,1], n>2$, is given by

$$
B_{W}\left(x_{1}, \ldots, x_{n}\right)=\left(\operatorname{sign}\left(\Pi_{i=1}^{n} x_{i}\right)\right) \max \left(0, \sum x_{i}-n+1\right) .
$$

Note that several construction methods for bipolar semicopulas were proposed in [10].

\section{Bipolar quasi-copulas and copulas}

In this section, we extend the notion of quasi-copulas and copulas acting on the unipolar scale $[0,1]$ to the bipolar scale $[-1,1]$.

Definition 8 Let $B \in \mathcal{B P}$ be 1-Lipschitz, then $B$ is called a bipolar quasi-copula.

Based on theorem 1 we have the following result.

Corollary $2 B \in \mathcal{G P}$ is a bipolar quasi-copula if and only if $B=\left(Q_{1}, Q_{2}, Q_{3}, Q_{4}\right) \in$ $\mathcal{Q}^{4}$.

Evidently, each bipolar quasi-copula is also a bipolar semicopula.

Definition 9 Let $B \in \mathcal{G P}$ has a bipolar neutral element $e=1$ and let $B$ be supermodular, then $B$ is called a bipolar copula. 
Corollary $3 B \in \mathcal{G P}$ is a bipolar copula if and only if $B=\left(C_{1}, C_{2}, C_{3}, C_{4}\right) \in \mathcal{C}^{4}$.

Observe that each bipolar copula $B$ is also a bipolar quasi-copula, and that the class of all bipolar quasi-copulas $\mathcal{B Q}$ is a sup - (inf - ) closure of the class $\mathcal{B C}$ of all bipolar copulas. $\Pi$ and $B_{M}$ are typical example of simple bipolar copulas.

As an example of a bipolar copula $B$ which is not simple, we consider the function $B:[-1,1]^{2} \rightarrow[-1,1]$ given by

$$
B(x, y)=x y+|x y|(1-|x|)(1-|y|) .
$$

Then $B=\left(C_{1}, C_{2}, C_{1}, C_{2}\right)$ where $C_{1}, C_{2} \in \mathcal{C}$ are Farlie-Gumbel-Morgenstern copulas [18] given by

$$
C_{1}(x, y)=x y+x y(1-x)(1-y)
$$

and

$$
C_{2}(x, y)=x y-x y(1-x)(1-y)
$$

\section{Generalized bipolar sum}

Sum $S$ on bipolar scale is defined on $\mathbb{R}^{2}$, and if restricted to domain $[-1,1]^{2}$ and truncated in the range to $[-1,1]$, we call it bipolar truncated sum BTS: $[-1,1]^{2} \rightarrow[-1,1]$,

$$
B T S(x, y)= \begin{cases}1 & \text { if } x+y>1 \\ x+y & \text { if }|x+y| \leq 1 \\ -1 & \text { if } x+y<-1\end{cases}
$$

The restriction of BTS to $[0,1]$ is standard truncated sum on $[0,1]$ (Lukasiewicz t-conorm).

The BTS is characterized by standard increasingness and by the fact that 0 is its neutral element.

Definition 10 A function $K:[-1,1]^{2} \rightarrow[-1,1]$ is a generalized bipolar sum (GBS) whenever it is increasing in both coordinates, it has 0 as neutral element (i.e. $K(x, 0)=$ $K(0, x)=x$, for each $x \in[-1,1])$ and $\left.K\right|_{\{-1,0,1\}^{2}}=\left.B T S\right|_{\{-1,0,1\}^{2}}$.

Distinguished examples of GBSs are the BTS and the symmetric maximum of Grabisch [5], $x \otimes y=\operatorname{sign}(x+y) \cdot(|x| \vee|y|)$.

By definition, a GBS is non associative, indeed suppose that $C$ is an associative GBS, then $0=C(1,-1)=C(C(1,1),-1)=C(1, C(1,-1))=C(1,0)=1$, which is a contradiction. Due to absence of associativity, GBSs differ from uninorms on $[-1,1]$ with neutral element $e=0$.

Note that, in general, we are not able to elicit a corresponding (simple) GBS from any generalized sum $S_{g}$ on $[0,1]$ (which is an aggregation function with neutral element 0 , i.e., $S_{g}$ is a dual semicopula) by way of

$$
K_{S_{g}}(x, y)=\operatorname{sign}(x+y) \cdot S_{g}(|x|,|y|)
$$


Indeed, equation (10) works only if $S_{g}=\max$ and in this case $K_{\max }$ is the Grabisch symmetric maximum $\otimes$. In any other case, the monotonicity of $K_{S_{g}}$ is violated. Consider, e.g., the Lukasiewicz t-conorm $L$, then $K_{L}$ does not coincide with the BTS (see figure 1) and, moreover it is not a GBS, due to absence of increasingness: $K_{L}(-.3, .4)=.7>K_{L}(.2, .4)=.6$. Note that the function $K_{S_{g}}$ given by (10) can be seen as a bipolar symmetrization of a dual semicopula $S_{g}$.

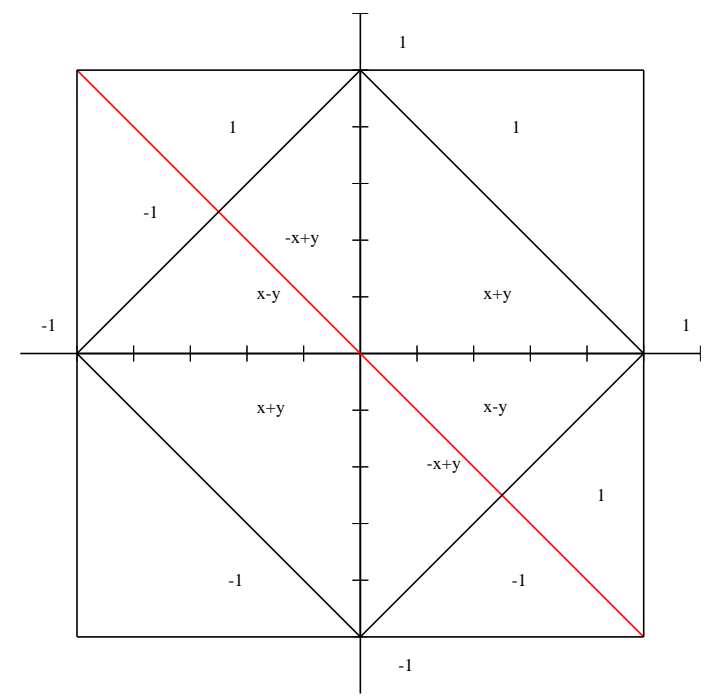

Fig. 1. $K_{L}(x, y)=\operatorname{sign}(x+y) \cdot L(|x|,|y|)$, being $L$ the truncated sum on $[0,1]$.

Now we describe some methods to generate GBSs. Firstly, we consider a convention of symmetry for addition on extended real line, i.e., $+\infty+(-\infty)=0$, then for any increasing bijection $f:[-1,1] \rightarrow[-\infty, \infty]$ such that $f(0)=0$, one can introduce the following (generated) GBS

$$
K_{f}(x, y)=f^{-1}(f(x)+f(y)) .
$$

Consider, e.g., $f(x)=\ln \frac{1+x}{1-x}$, then $f^{-1}(x)=\frac{e^{x}-1}{e^{x}+1}$ and $K_{f}(x, y)=\frac{x+y}{1+x y}$, which is bipolar Einstein sum.

Let us consider an increasing function $f:[-1,1] \rightarrow[-\infty, \infty]$ such that $f(0)=0$ and $f(-1)=-f(1)$. We can generate the following GBS (with convention $+\infty+$ $(-\infty)=0$ if necessary)

$$
K_{f}(x, y)=f^{-1}(\min \{f(1), \max \{f(-1), f(x)+f(y)\}\}) .
$$

Now BTS is generated by formula (12) using identity $f(x)=x$. For power $p>0$, also $f_{p}(x)=\operatorname{sign}(f(x)) \cdot|f(x)|^{p}$ generates a BTS, $K_{f_{p}}$, so we have a parametric 
family of generated GBSs. If $f$ is odd (this is not necessary, in general) then pointwise limit for $p \rightarrow \infty$ is just $\emptyset$.

Also for GBS a representation theorem could be formulated.

Theorem $3 K:[-1,1]^{2} \rightarrow[-1,1]$ is a GBS iff there is quadruple $\left(A_{1}, B_{1}, A_{2}, B_{2}\right)$ with 4 Aggregation functions on $[0,1]^{2}, A_{1}$ and $A_{2}$ coinciding with truncated sum on boundaries (i.e., dual semicopulas), $B_{1}, B_{2}$ coinciding with arithmetic mean on boundaries connecting points $(0,0),(1,0)$ and $(1,1)$, and on point $(0,1)$, such that

$$
K(x, y)=\left\{\begin{array}{lll}
A_{1}(x, y) & \text { if } \quad(x, y) \in[0,1]^{2} \\
2 B_{1}(x+1, y)-1 & \text { if } \quad(x, y) \in[-1,0] \times[0,1] \\
-A_{2}(-x,-y) & \text { if } \quad(x, y) \in[-1,0]^{2} \\
2 B_{2}(x, y+1)-1 & \text { if } \quad(x, y) \in[0,1] \times[-1,0]
\end{array}\right.
$$

We conclude the paper with some considerations linking GBS and GBP. For any two additive generators $f$ and $g, g$ bounded, of GBS, put $D(x, y)=f^{-1}\left(\frac{g(x) \cdot f(y)}{g(1)}\right)$, then $D$ is a GBP. E.g. putting $g(x)=x$, one obtains bipolar multiplication of Weber given by $f^{-1}(x \cdot f(y))$ (in unipolar situation it is background of Weber integral [22], our bipolar can serve for bipolar Weber integral).

For any additive generator $f$ of GBS, $f$ restricted to $[0,1]$ is an additive generator of an Archimedean t-conorm, it is our $A_{1}$ in quadruple representation, (and similarly, $f(-x)$ restricted to $[0,1]$ is an additive generator of an Archimedean t-conorm $A_{2}$. Weber integral is based on such a t-conorm and related unipolar generalized product, and its bipolar extension would then be based on generalized bipolar sum (generated by $f$ ) and the corresponding bipolar generalized product.

\section{Concluding remarks}

We have introduced and discussed bipolar generalizations of the product, including bipolar semicopulas, bipolar triangular norms, bipolar quasi-copulas and bipolar copulas. We have also considered bipolar generalizations of the truncated sum, having the symmetric maximum of Grabisch as distinctive element. Stress that the difference from nullnorms $(\mathrm{a}=0)$ and uninorms $(\mathrm{e}=0)$ on $[-1,1]$ are obvious, due to the absence of associativity. Observe that our approach to bipolar aggregation can be seen as a particular case of the multi-polar aggregation proposal as given in [17] for dimension $n=2$. Though there is a minor overlap with conjunctive aggregation functions in the area of Atanassov's intuitionistic framework, our concept is rather different (especially, the bipolar neutral element makes the difference). Observe that our approach brings a generalization of the product (considered as a multiplication for integrals) into the bipolar framework, where it is supposed to play the role of multiplication when constructing integrals. We expect application of our results in multicriteria decision support when considering bipolar scales, especially when dealing with bipolar capacities based integrals. Observe that simple bipolar semicopulas were already applied when introducing universal integrals on the bipolar scale $[-1,1]$, see [8]. 


\section{Acknowledgment}

The work of R. Mesiar on this paper was supported by the European Regional Development Fund in the IT4Innovations Centre of Excellence project reg. no. CZ.1.05/1.1.00/ 02.0070, and the grant VEGA 1/0420/15.

\section{References}

1. B. Bassan and F. Spizzichino. Relations among univariate aging, bivariate aging and dependence for exchangeable lifetimes. Journal of Multivariate Analysis, 93(2):313-339, 2005.

2. G. Beliakov, A. Pradera, and T. Calvo. Aggregation functions: A guide for practitioners. Studies in Fuziness and Soft Computing, Springer, Berlin, 2008.

3. D. Dubois and H. Prade. An introduction to bipolar representations of information and preference. International Journal of Intelligent Systems, 23(8):866-877, 2008.

4. F. Durante and C. Sempi. Semicopulæ. Kybernetika, 41(3):315-328, 2005.

5. M. Grabisch. The symmetric Sugeno integral. Fuzzy Sets and Systems, 139(3):473-490, 2003.

6. M. Grabisch, S. Greco, and M. Pirlot. Bipolar and bivariate models in multicriteria decision analysis: Descriptive and constructive approaches. Int. J. Intell. Syst., 23(9):930-969, 2008.

7. M. Grabisch, J.L. Marichal, R. Mesiar, and E. Pap. Aggregation Functions (Encyclopedia of Mathematics and its Applications). Cambridge University Press, 2009.

8. S. Greco, R. Mesiar, and F. Rindone. The Bipolar Universal Integral. In IPMU 2012, Part III, CCIS 300, pages 360-369. Greco et al, 2012.

9. S. Greco, R. Mesiar, and F. Rindone. Generalized Product. In IPMU 2014, CCIS 444, pages 289-295. Springer International Publishing, 2014.

10. S. Greco, R. Mesiar, and F. Rindone. Bipolar semicopulas. Fuzzy Sets and Systems, 268(0):141 - 148, 2015.

11. S. Greco and F. Rindone. Bipolar fuzzy integrals. Fuzzy Sets and Systems, 220:21-33, 2013.

12. S. Greco and F. Rindone. The bipolar Choquet integral representation. Theory and Decision, 77(1):1-29, 2014.

13. P. Hájek. Metamathematics of fuzzy logic, volume 4. Springer, 1998.

14. D. Kahneman and A. Tversky. Prospect theory: An analysis of decision under risk. Econometrica: Journal of the Econometric Society, 47(2):263-291, 1979.

15. E. P. Klement and E. Mesiar, R. Pap. Triangular norms. Dordrecht, The Netherlands: Kluwer, 2000.

16. E.P. Klement, R. Mesiar, and E. Pap. A universal integral as common frame for Choquet and Sugeno integral. IEEE Transactions on Fuzzy Systems, 18(1):178-187, 2010.

17. A. Mesiarová-Zemánková and K. Ahmad. Multi-polar Choquet integral. Fuzzy Sets and Systems, 220:1-20, 2013.

18. R. B. Nelsen. An introduction to copulas. Springer, New York, 2006.

19. B. Schweizer and A. Sklar. Statistical metric spaces. Pacific J. Math., 10:313-334, 1960.

20. A. Tversky and D. Kahneman. Advances in prospect theory: Cumulative representation of uncertainty. Journal of Risk and uncertainty, 5(4):297-323, 1992.

21. Z. Wang and G. J. Klir. Generalized measure theory, volume 25. NewYork: Springer-Verlag, 2009.

22. S. Weber. $\perp$-decomposable measures and integrals for archimedean t-conorms $\perp$. Journal of Mathematical Analysis and Applications, 101(1):114-138, 1984. 